\title{
Especies clínicas comunes del género Scopulariopsis Bainier y taxas relacionados
}

\author{
(Common clinical species of the Scopulariopsis Bainier genera and \\ related taxa)
}

Eduardo Piontelli, L

Prof. Emérito Cátedra de Micología, Universidad de Valparaíso Casilla 92V Valparaíso (Chile).

\section{RESUMEN}

Los integrantes del género Microascus y su anamorfo Scopulariopsis, incluyen especies fúngicas comunes del suelo, excrementos, ambientes internos y algunas reconocidas como oportunistas en los animales, incluyendo al hombre. La sola morfología del género no siempre permite un buen diagnóstico a pesar de su utilidad cuando se presentan los anamorfos o teleomorfos o ambos. Sin embargo, la biología molecular ha permitido el análisis de muchos aislados clínicos, contribuyendo con nuevos aportes y nuevas combinaciones taxonómicas en la literatura actual. Este trabajo, reune lo más actual sobre estos géneros comunes en salud pública, aportando claves morfofisiológicas útiles para su reconocimiento primario en los laboratorios clínicos de baja complejidad.

\section{INTRODUCCIÓN}

El género Scopulariopsis (asexual) fue erigido por Bainier (1907) con la especie S. brevicaulis como tipo. Anteriormente, $\boldsymbol{S}$. brevicaulis fue descrito originalmente como Penicillium brevicaule por Saccardo (1882) un integrante de la antigua sección Anomala de Penicillium (Biourge 1923). Actualmente las estructuras que permiten distinguir el género son su conidiogénesis anelídica, la gruesa pared de los conidios, la base trunca de estos que se disponen en largas cadenas secas y el color de sus colonias que varía desde el blanco al café oscuro, pero nunca en tonalidades del verde como los Penicillium (Barron et al, 1961; Morton \& Smith 1963, Sandoval-Denis et al, 2013-2015).

Mediante cultivos, cruzamientos y métodos moleculares se ha demostrado a lo largo de la literatura que el estado sexual de Scopulariopsis pertenece al género Microascus (Ascomycota, Microascales, Microascaceae, Curzi, 1931, Abbott et al. 1998, Issakainen et al. 2003; Lumbsch \& Huhndorf 2007). Abbott \& Sigler (2001), confirmaron la existencia de especie homotálicas y heterotálicas de Microascus, el cual se caracteriza por tener ascomata periteciales con cuellos papilados o cilíndricos, ascos ovados a globosos, unitunicados, no pedicelados y evanescentes con 8 ascosporas unicelulares, típicamente asimétricas, reniformes, lunadas o triangulares. Las ascosporas se liberan en largos cirros por el ostiolo o como una pelota gelatinosa

\begin{abstract}
Members of the genus Microascus and their anamorph Scopulariopsis, include common fungal species of soil, droppings, indoor environments and some recognized opportunistic in animals, including humans. The morphology of the genus alone is not always sufficient for a proper diagnosis despite its usefulness when anamorphs or teleomorphs or both are present. However, molecular biology has allowed the analysis of many clinical isolates, contributing with new imput and new taxonomic combinations in the current literature. This work brings together the latest on these genera in pubblic health, providing a primary key for useful morphophysiological determination in clinical laboratory of low complexity.
\end{abstract}

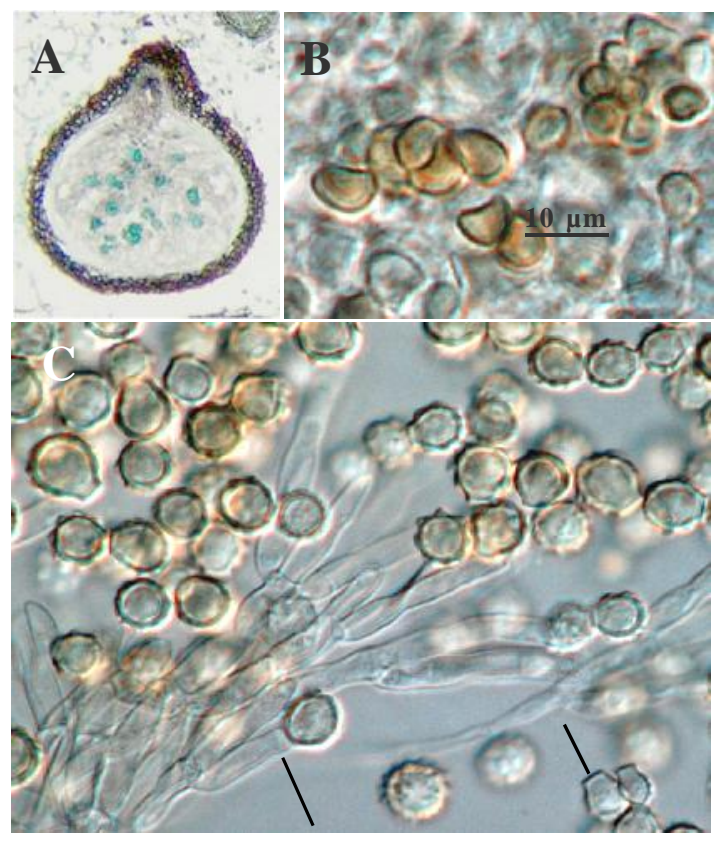

Figura 1. A-B-C. A . Peritecio de Microascus sp. con su hamatecio (Tomado de: path.upmc.edu.case 135/dx. html) BC. Microascus brevicaulis. B. Ascosporas lunadas, C. S. brevicaulis anamorfo, conidióforos, locus conidiógeno proliferante (flecha) conidios de base trunca (flecha). 
en el apex del ascomata (Morton \& Smith 1963).

Análisis filogenético. A pesar que el género Pithoascus (Microascaceae) nominado por Von Arx (1973), es considerado sinónimo de Microascus por varios autores (entre ellos Guarro et al, 2012),algunas especies muestran características morfológicas intermedias con Microascus. Sandoval-Denis et al, (2015), vuelven a aceptar Pithoascus y erigieron además el nuevo género no patogénico en mamíferos (por no crecer a $30^{\circ} \mathrm{C}$ ), Pseudoscopulariopsis (tipificado por P.shumacheri) mediante un gran set de aislamientos y el empleo de datos de secuencias de DNA de 4 locus (región de ITS, fragmentos de DNAr LSU, el factor de traducciónelongación 1 alfa y la beta tubulina). Se describen además 7 nuevas especies de Microascus y 1 de Scopulariopsis. Los datos filogenéticos muestran que Scopulariopsis/Microascus son polifiléticos con especies distribuidas en distantes linajes, sin embargo, muchas especies de ambos taxas se agrupan en un único y bien definido linaje que comprende 4 sublinajes que Sandoval-Denis et al, (2015) interpretan como 3 distintos géneros: Microascus, Scopulariopsis y Pithoascus, además de un cuarto que representa un género aún no descrito.

Ecología. Scopulariopsis incluía unas 25 especies, sin embargo, actualmente su número es más reducido debido a que muchas de éstas son dudosas y se han excluido del género por no ser filogenéticamente asociadas o no poseer un extipo (Issakainen et al, 2003; Sandoval-Denis, 2015). Su distribución mayoritaria es generalmente cosmopolita y se aislan comunmente desde el suelo, aire, detritus orgánicos diversos, la vegetación y excrementos de herbívoros (Domsch et al. 2007). Sin embargo, algunas especies se han descrito como colonizadores o patógenas de mamíferos, incluyendo humanos e insectos (deHoog et al, 2000, Iwen et al, 2012, Sandoval-Denis et al, 2013).

Acorde al nuevo Código Internacional de Nomenclatura para los hongos algas y plantas, en los hongos como en otros microorganismos debe emplearse un solo nombre ya sea el anamorfo o el teleomorfo según corresponda (Hawksworth, 2012). Según Sandoval-Denis et al, (2015), el nombre de Scopulariopsis se ha usado más frecuentemente en la literarura (incluyendo la médica) y debe tener prioridad sobre Microascus, a pesar que aún no se ha propuesto por los especialistas internacionales. Debido a ésto, aplicaremos aquí también los nombres de las especies de Scopulariopsis y Microascus por separado.

A pesar que la sola morfología de Scopulariopsis/ Microascus y algunos datos fisiológicos permiten un buen acercamiento a la especie, ciertos caracteres se sobreponen en el morfotipo en algunos, sin embargo, debemos resaltar que las diferencias morfológicas más utiles para distinguir estos géneros, las más informativas se correlacionan con los datos fillogenéticos, tales como la forma, tamaño y color de las anélides, forma de las ascosporas y la presencia de un poro germinativo, no así la forma y tamaño del ascomata y el tamaño y forma del cuello ostiolar, que se asocian frecuentemente con las condiciones del cultivo, cambios ambientales e incubación (Barron et al. 1961).
Un significante problema de salud pública en el siglo 19 (no del todo esclarecido), se relacionó a situaciones de ambientes internos, donde los habitantes de muchas casas decoraban sus paredes con papeles murales que contenían pigmentos arsenicales verdes. El problema al parecer, era causado por ciertos microorga-nismos, en especial algunos hongos como S. brevicaulis que crecian en presencia de arsenico inorganico para formar un gas toxico con olor a ajo; el fatal gas volatil trimetilarsina (gas Gosio). Se aconseja leer al respecto el detallado trabajo de Bentley \& Chasteen (2002).

Patología: Las especies clínicas más frecuentes del género reportadas en las diferentes localizaciones anatómicas en la liteartura no sobrepasan las 10 especies: entre ellas la más frecuente es: Scopulariopsis brevicaulis, seguida de S.cordiae, S.brumptii, S. flava, Microascus (Scopulariopsis) niger, M. cinereus, M.alveolaris, M.cirrosus, M.manginii y M.trigonosporus (de Hoog et al, 2000; Gluck et al, 2011; Sandoval-Denis et al, 2013). Principalmente causan infecciones superficiales y representando además una de las causas comunes de onicomicosis no dermatofítica (de Hoog et al, 2000). Otras manifestaciones poco frecuentes son: queratitis debido a trauma ocular (Ragge et al, 1990), otomicosis (Hennequin et al, 1994; Besbes et al. 2002), así como en tejidos profundos diversos en pacientes con compromiso inmune (Patel et al,1993; Petit et al, 2011), pero también en individuos inmunocompetentes(Kriesel et al, 1994; Migrino et al, 1995). La literatura destaca que la mayoría de los aislados clínicos muestran altos niveles de resistencia frente a los agentes antifúngicos comunes (Aguilar et al, 1999; Miossec et al, 2011; Sandoval-Denis et al 2013).

Morfología del género Scopulariopsis. Las colonias crecen rápidamente, varían en color desde el blanco, crema, gris, piel de ante, de color marrón, negro o son predominantemente de color marrón claro. La morfología microscópica muestra cadenas de conidios unicelulares (ameroconidios) producidos en forma basipeta (donde el conidio más joven está en el extremo basal de la cadena) por una célula conidiógena especializada llamada anélide. En Scopulariopsis, las anélides

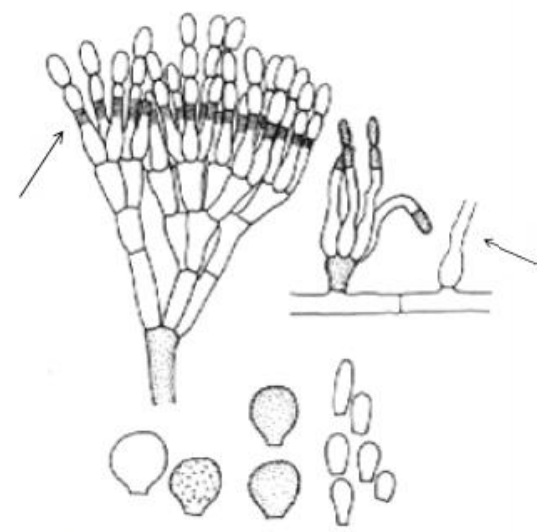

Figura 2. Scopulariopsis spp. mostrando conidióforo, células conidiógenas en racimo o solitarias con anélides (flechas) y tipos de conidios (Tomado de: http://website.nbm-mnb.ca/ mycologywebpages/Moulds/Scopulariopsis.html 
pueden ser solitarias, en grupos, semejando a una forma de Penicillium (Por anélide se entiende una sucesión de conidios blásticos, medinte percurrentes proliferaciones de la célula coni-diógena. La punta de una anélide aumenta en longitud y se hace más estrecha cuando se forma cada conidio posterior (Vea fig. 2). Los conidios son globosos a piriformes, lisos o rugosos y de base trunca (Fig. 1-2).

Morfología del género teleomorfo Microascus

Colonias restringidas o esparcidas, de color gris pálido, café, oliváceo o negro, flocosas o fasciculadas, granulares o formando anillos concéntricos debido a la producción de los ascomata. Ascomata periteciales o raramente no ostiolados, inmersos o erumpentes, esféricos a ampuliformes, con un cuello cilíndrico a veces largos y setoso, ocasionalmente como papila. Peridio café oscuro, compuesto por células de paredes gruesas de texura angularis o intricata. Ascos unitunicados, con 8 esporas, abovadas en forma de barril o esféricas, evanescentes usualmente formadas en grupos basales. Ascosporas unicelulares, reniformes, en forma de corazón o angulares en visión lateral, dextrinoides cuando jóvenes de color paja al madurar, lisas, a veces con un incospicuo poro germinal, que se liberan al exterior al madurar en forma de una gota gelatinosa en el ápice del ascoma o en forma de un largo cirro (Sandoval-Denis et al, 2015).

Medios de cultivo. Son de utilidad, el agar avena y el agar papa zanahoria. Ambos se cultivan a diferentes temperaturas principalmente $\left(5,25,30,35,40^{\circ} \mathrm{C}\right)$.

Clave morfológica que permite separar Microascus,

\author{
Scopulariopsis y géneros aliados \\ (Tomada de Sandoval-Denis et al, 2015)
}

1. -Colonias blancas, canela o café;células conidiógenas cilíndricas, hialinas o café pálidas; conidios de paredes gruesas con una protuberante base plana ................................. Scopulariopsis -Colonias grises-blancas, oliva-verdes o negras; células conidiógenas ampuliformes a lageniformes, subhialinas o café-verdes, conidios de otro tipo 2

2. -Morfo asexual ausente;si está presente, conidióforos, cortos, únicos; Ascosporas sin poro germinal 3 -Morfo asexual siempre presente, conidióforos a menudo ramificados hasta 80 um de largo; ascosporas con poro germinal Microascus

3. -Ascomata con un peridio de textura angularis; morfo asexual cuando presente que forma anélides cortas únicas, hialinas Pithoascus -Ascomata con un peridio con células de texura epidermoidea; morfo asexual usualmente abundante, que forma largas anélides desde cortos e hinchados conidióforos que se oscurecen con la edad (saprotrofo) ......... Pseudoscopulariopsis

\section{Clave de las especies clínicas comunes de Scopulariopsis}

(Modificada de Sandoval-Denis et al, 2015)

1. Colonias blancas con tendencia a gris claro cuando los ascomata están presentes

- Colonias canela, café pálido a café fusco.

2. - Conidios lisos

- Conidios rugosos en la madurez

3.

- Conidióforos abundantes; conidios 6-9,5 x 5-8,5 $\mu \mathrm{m}$; ascosporas reniformes o con forma de corazón. Conidióforos, cuando presentes, hialinos. (crece $\mathbf{a} 35^{\circ} \mathrm{C}$ ).

S. candida(Gueguen) Vuill.

( Es una especie común en el hemisferio norte ya sea en el suelo, aire, polvo como también de tejidos superficiales en humanos y animales (de Hoog et al, 2011). Se diferencia de S. brevicaulis, S.asperula y S.flava por sus conidios hialinos, subglobosos a ampliamente ovados y lisos. Cuando el teleomorfo está presente (M.manginii (Loubiere) Curzi, se caracteriza por sus globosos peritecios 100-170 $\mu$ m diám. y ascosporas reniformes a forma de corazón que son algo más anchas ( 4-6 x 5-6 $\mu \mathrm{m}$ ) que las de sus relativos cercanos $S$. brevicaulis (5-6 x 3,5-4,5 $\mu \mathrm{m})$ S.cordiae (4,5- 5,5 x 3,5-4 $\mu \mathrm{m})$ y S.soppii (6-7 x2,5-3 $\mu \mathrm{m}$ ). Figuras: tomadas de: Matsushima, T. (1975).

- Conidióforos escasos; conidios 2,5 - 6 x 2-5 $\mu \mathrm{m}$.; ascosporas color paja, reniforme a ampliamente lunadas $\left(\right.$ Crece a $\left.40^{\circ} \mathrm{C}\right)$... S.cordiae Sandoval-Denis, Gené \& Cano sp. nov.

(Colonias en agar avena y PCA de 35-36 y 48-50 mm diám., a los 14 días de incubación, planas con escaso micelio aéreo, blancas a color gris claro, de aspecto granular por la abundante producción de ascoma, reverso blancuzco. Ascomata abundantes, negros, lisos, superficiales o inmersos, globosos a sub-

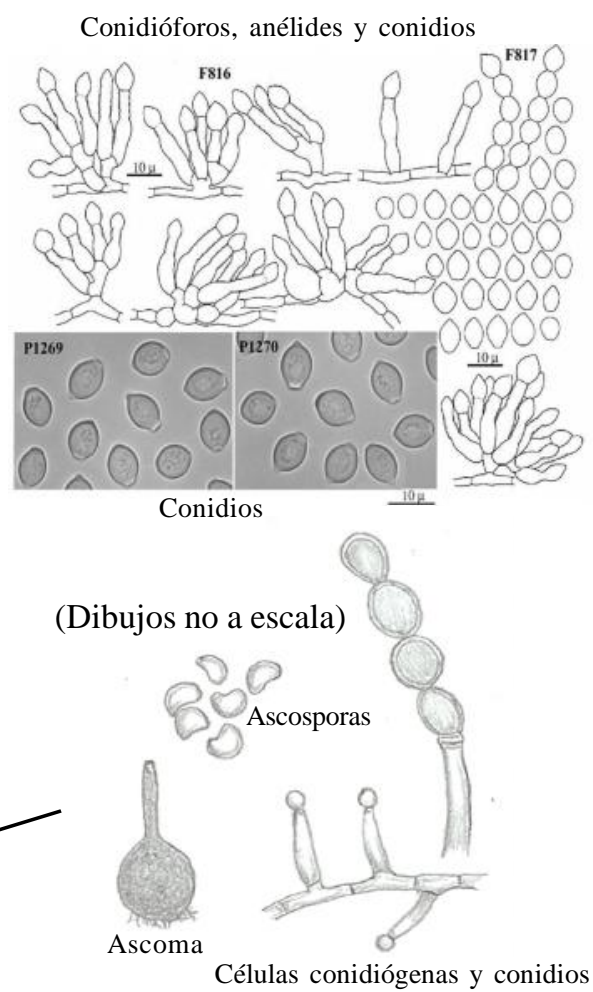


globosos, 100-150 $\mu$ m diám., con un largo cuello cilíndrico ostiolado, de hasta unos $390 \mu \mathrm{m}$, con peridio de textura angularis. Ascos irregulares, elipsoidales 9-15 xt,5-10 $\mu \mathrm{m}$. Ascosporas ampliamente lunadas a reniformes, 4,5-5,5 x 3,5-4 $\mu \mathrm{m}$, color paja y amarillas en masa con un único e incospicuo poro germinal. Conidióforos ausentes, anélides sésiles, que nacen únicas y lateralmente en las hifas vegetativas, hialinas, lisas de paredes delgadas, cilíndricas 8-15 x 1,5-3,5 $\mu \mathrm{m}$, conidios ampliamen te elipsoidales de base trunca, hialinos en masa, lisos y de paredes gruesas, arreglados en cadenas. Clamidosporas no observadas. Máxima temperatura $40^{\circ} \mathrm{C}$, mínima $15^{\circ} \mathrm{C}$. S. cordiae en su fase sexual, semeja morfológicamente al teleomorfo de S.candida en la forma y tamaño del ascomata, ascos y ascosporas. Se diferencia por su rápido creci miento, la brillante aparencia de las colonias, la presencia de un largo cuello cilíndrico en los ascomata sumergidos y su reducido tamaño de las ascosporas y conidios (Sandoval-Denis et al, 2015).

4. - Conidios de color blanco en masa, globosos a obovoidales, 6, 5 7 x 5,5- 6,5 $\mu \mathrm{m}$, teleomorfo no observado (crece a $35^{\circ} \mathrm{C}$ ).... S.flava F.J.Morton \& G. Smith.

(Es una especie que se aisla del queso, suelo, insectos y como oportu nista en humanos (De Hoog et al, 2011, Piontelli \&Toro 1988). Es una especie cercana a S.brevicaulis, sin embargo, este produce colonias canela pulverulentas a granulosas con conidios globosos a ovoides con ápice redondeado o en punta, que se torna verrugoso en el tiempo y café pálido. S.flava, produce colonias blancas flocosas a fasciculadas y conidios globosos aobovoides hialinos con ápices redondeados y toscamente rugosos. Filogenéticamente $S$.flava se relaciona con S.soppii, pero este último difiere en la producción de grandes conidios $(5,5-9$ x 5-8 $\mu \mathrm{m}$, versus 6,5-7x 5,5-6,5 $\mu \mathrm{m})$. El teleomorfo de S.flava no se ha reportado en el tiempo desde el trabajo de Sopp,1912).Figura tomada de: deHoog et al, 2000. -Conidios de otro color. 5

Conidióforos, anélides y conidios

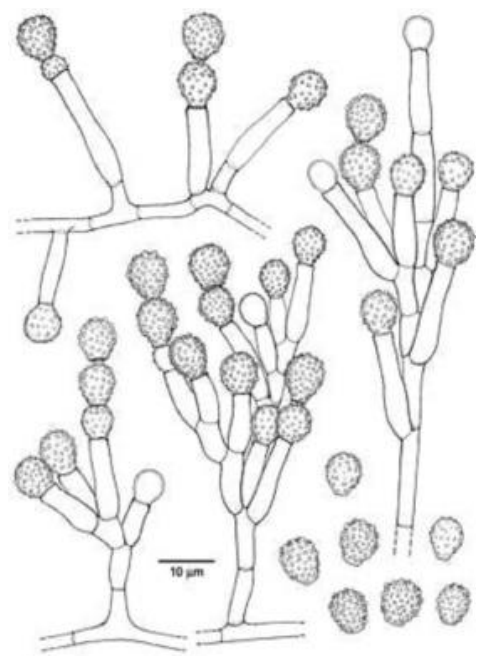

5. - Conidios café en masas, verrugosos en su madurez, globosos a ovoides; 6-9 x 5,5,-9 $\mu \mathrm{m}$................ S. brevicaulis (Sacc.) Bainier (sinónimo básico = Penicillium brevicaulis Sacc.)

(Es una especie cosmopolita aislada de un amplio rango de sustratos tales como: el suelo,madera , paja, granos, fruta, insectos muertos, excrementos de herbívoros, papel, pelaje de animales diversos, carnes quesos, leche etc. y como patógeno oportunista en humanos(de Hoog etal, 2011; Sandoval-Denis et al, 2013) Teleomorfo =M.brivicaulis (Ab-bott et al, 1998). Colonias de crecimiento relativamente rápido, de color avellana a café. Células conidiógenas en pequeños grupos penicilados, en hifas indiferenciadas, cilíndricas con base ligeramente dilatada, 9-25 x 2,5-3,5 $\mu \mathrm{m}$, con zonas aneladas de igual ancho y largo variable. Conidios subhialinos, esféricos a ovoides o en forma de bala (mitral), de base trunca mayoritariamente de paredes rugosas, crece a $27^{\circ} \mathrm{C}$ como temperatura máxima, teleomorfo cuando presente, de cuellos cortos, negros 80-150 x 70-130 $\mu \mathrm{m}$, peridio seudoparenquimatoso, 8 esporas por ascos, subesféricos, 8$10 \mu \mathrm{m}$ en diám., evanescentes. Ascosporas ampliamente reniformes, 5-6 x 3,5-4,5 $\mu \mathrm{m}$, lisas, naranja en masas: la presencia del teleomorfo es muy rara en los cultivos heterotálicos.

-Conidios de color café grisáceo en masas, redondos y ápices redondeados, rugosos, 3,5-4,5 de ancho ......S. brumptii Sal- Duval (Teleomor. Microascus paisii (Pollacci) Sandoval-Denis, Gené \& Guarro)

(Colonias de crecimiento moderado, blancas al principio, luego
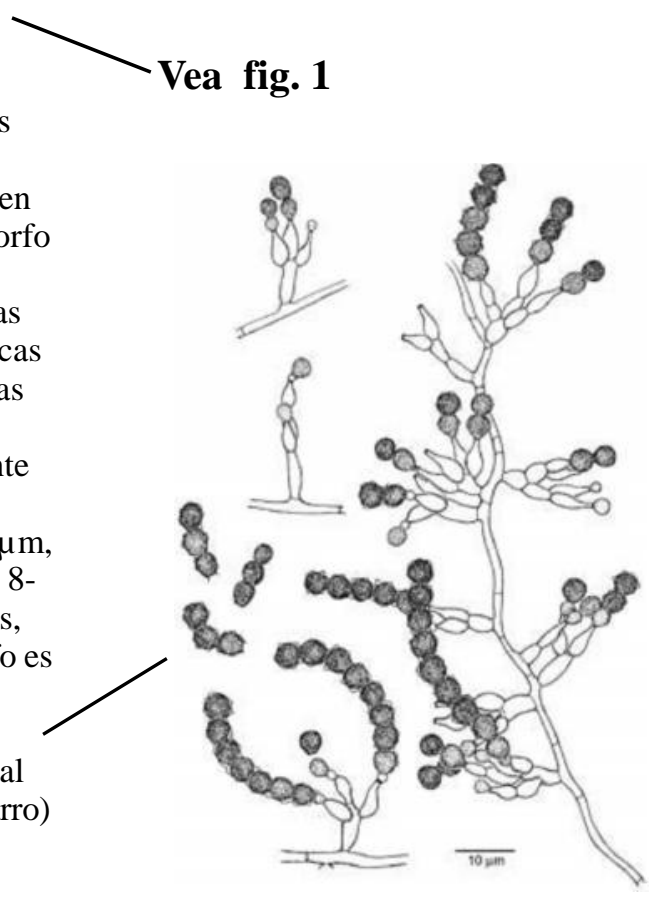

Bol. Micol. 2015; 30(1): 27 - 33

micologia.uv.cl 
grises, finalmente sepia gris a cafesosas o en pequeños grupos semejante a pinceles, de base dilatada que se adelgazan hacia el ápice. Células conidiógenas solitarias en hifas indiferenciafas 5-10 x 2,5-3,5 $\mu \mathrm{m}$, con zonas aneladas cilíndricas de largo variable, incospicuas. Conidios café oscuros, obovoides de base trunca, $4-5,5$ x 3,5-4,5 $\mu \mathrm{m}$, de paredes rugosas. La nueva combinación teleomórfica para $S$. brumptii, llamada Microascus paisii (Plollacci) Sandoval-Denis, Gené \& Guarro, es la que corresponde debido a su confusa historia nomenclatural. Se diferencia de especies cercanas como $\boldsymbol{M}$. chartarus y $\mathbf{M}$. croci por sus colonias oscuras y su capacidad de esporular a $\left.37^{\circ} \mathrm{C}\right)$. Figura tomada de: de Hoog et al, (2000).

- Conidios café oscuros a fusco, lisos o verrugosos, globosos a ovados, usualmente con un ápice en punta, 5 - 8 x 4 - 6,5 $\mu \mathrm{m}$. (Sacc.) Hughes (= Microascus niger (Sopp)Curzi).

(Morfológicamente $\boldsymbol{S}$. asperula es cercano a S. brevicaulis y $\mathbf{S}$. flava,sin embargo, $\boldsymbol{S}$. asperula se diferencia por tener colonias de color café oscuro a fusco o violáceas y conidios globosos a ovados toscamente verrugosos o de paredes lisas, de color fusco a sepia, la mayoría con ápices en punta, en contraste, $S$. brevicaulis muestra colonia de color canela, conidios globosos y verrucosos, café pálidos un poco más grandes en medida, mientras $\boldsymbol{S}$. flava muestra colonias blancas y conidios verrugosos y hialinos más pequeños. $S$ asperula está en sinonimía con $\boldsymbol{S}$. fusca y $\boldsymbol{S}$. bestae (Ropars et al, 2012). Especie común en suelo aire, ambientes internos, quesos, mantequilla y como oportunista en humanos en uñas y piel). (Ropars et al, 2012; Sandoval -Denis et al, 2015. Crece a $35^{\circ} \mathrm{C}$ ). Figura tomada de: de Hoog et al, (2000).

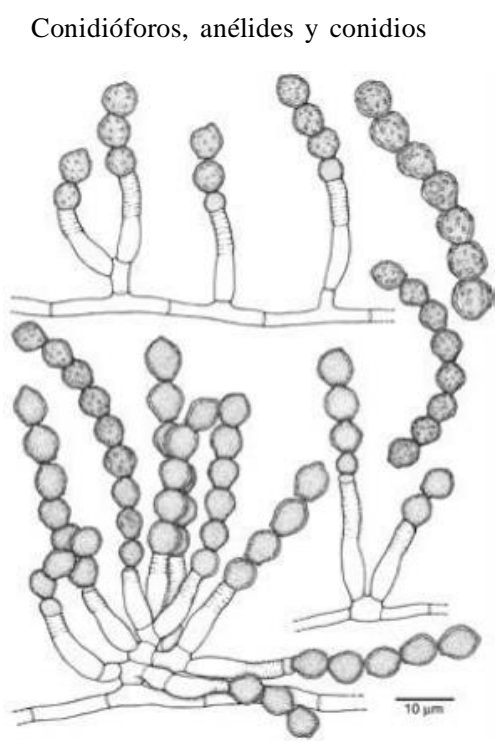

\section{Claves de las especies clínicas comunes de Microascus}

(Adaptada de Sandoval-Denis et al, 2015)

1. -Ascomata presente en cultivos 2

- Ascomata ausente en cultivos . (Vea: S. brumptii)

2. - Crecimiento a $40^{\circ} \mathrm{C}$ 3

-Sin crecimiento a $40^{\circ} \mathrm{C}$. 5

3. -Peridio de textura angularis, ascosporas siempre triangulares o quadrangulares estrechas, 4-6 x 2,5-4 $\mu \mathrm{m}$, amarillas en masa M.alveolaris Sandoval-Denis, Gené \& Guarro sp. nov. (Fig.3)

(Colonia en agar avena y PCA, de 31-36 y de 18-29 mm en 14 días a $25^{\circ}$, planas, aterciopeladas, centro de apariencia granular debido a la presencia de ascomata, reverso blaco a gris. Ascomata superficiales o inmersos globosos a subglobosos 110-290 $\mu \mathrm{m}$ diám., negros con hifas laxas dispersas, usualmente con un cuello cilíndrico de hasta $100 \mu \mathrm{m}$ de largo. Ascos irregularmente elipsoidales, 8-12 x 7,5- $11 \mu \mathrm{m}$. Ascosporas ampliamente triangulares, raramente reniformes, con un solo polo germinativo,4-6 x 3$5 \mu \mathrm{m}$, de color paja y amarillas en masa. Conidióforos ausentes o como únicas células basales que originan grupos de 2-3 anélides, raramente ligeramente ramificadas, hialinas a subhialinas, de paredes lisas. Anélides mayormente sésiles, únicas y laterales en hifas vegetativas, lageniformes 6-17 x 1,5-3,5 $\mu \mathrm{m}$ hialinas o subhialinas, lisas y de paredes delgadas. Conidios elipsoidales naviculares o en forma de bala, 3-5 x 2-3,5 $\mu \mathrm{m}$, con base trunca y ápice redondeado, subhialinos a café pálido, café en masa, naciendo lateral mente de las hifas, delgadas y lisas, arregladas en largas cadenas. Conidios solitarios a veces presentes sésiles o sobre un corto pie, 3,5 x 2,5-4 $\mu \mathrm{m}$, lisos y de pared más o menos gruesa, subhialinos o café pálido. Temp. óptima $25-30^{\circ} \mathrm{C}$ máxima $40^{\circ} \mathrm{C}$. Todas las cepas encontradas se aislaron del tracto respiratorio humano. Morfológicamente esta especie es similar a M.campaniformis, M.macrosporus, M.pyramidus y $\boldsymbol{M}$. trigonosporus por su forma de ascosporas, se diferencia por sus colonias membranosas y blancas, el pequeño tamaño de las ascosporas y los conidios angostos (Vea: Sandoval-Denis et al, 2015).

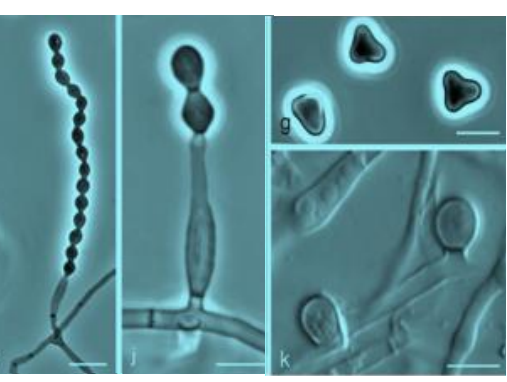

Fig. 3- i-j Conidióforos anélides y conidios, g, Ascosporas, k. Coni-dios solitarios. (Figuras tomadas de SandovalDenis et al, 2015) 
-Peridio de textura angularis, ascosporas reniformes a ampliamente lunadas, raramente triangulares, conidióforos usualmente únicos 4

4. -Ascomata hasta $300 \mu \mathrm{m}$ diám., ascosporas rojas café pálidas, conidios 2-3 $\mu \mathrm{m}$ de ancho M. cinereus (Émile-Weil \& Gaudin) Curzi.

Es una especie cosmopolita en amplios rangos de sustratos, frecuentemente de cereales, suelo y excremento de herbívoros (Guarro et al, 2012 ) pero también como oportunista en humanos y otros animales (Braddley et al, 2000; de Hoog et al, 2011; Sandoval-Denis et al, 2013). Colonias restringidas de color pálido a oscuro o negras grisáceas. Ascos de 7-12 x 5-10 $\mu \mathrm{m}$, con ascosporas reniformes de 4-5,5 x 2,5-4 $\mu \mathrm{m}$, con un incospicuo poro germinal; conidios amarillo claro,ampliamente clavados, de 3 $5 \times 2-3 \mu \mathrm{m}$, lunadas, de paredes lisas. Crece a $40^{\circ} \mathrm{C}$ ). Figura (parte) tomada de: Baddley et al, (2000).

-Ascomata menores que 230 um diám, ascosporas color paja, conidios 4-6 $\mu \mathrm{m}$ de ancho M.cirrosus Curzi

(Ascomata subesféricos con cuello relativamente largo y cilíndrico rodeado de pelos apicales 140-230 $\mu \mathrm{m}$ diám. Ascos obovides a esféricos con 8 ascosporas, 9-12 x 8- $11 \mu \mathrm{m}$. Es similar a M.cinereus; sin embargo, M.cirrosus presenta ascosporas ampliamente reniformes, de 5-6 x 4-6 $\mu \mathrm{m}$ y grandes conidios, subglobosos ovados en largas cadenas secas ampliamente clavados y de base trunca 4 - 6,5 x 4-6 $\mu$ m. Según Sandoval-Denis et al, (2015), la excepa tipo de Torula paisii (Scopulariopsis paisii) es filogenéticamente distante de la ex especie tipo de M.cirrosus y debe ser conside rada una especie distinta. $\boldsymbol{M}$. cirrosus puede distinguirse por tener conidios subglobosos a abovados de, 4-6,5 x 4-6 $\mu \mathrm{m}$, mientras los de $\boldsymbol{M}$. paisii son ampliamente elipsoidales a clavados cortos, que miden, 4-6 x 2-4,5 $\mu \mathrm{m}$. M.cirrosus es también similar a $\boldsymbol{M}$. cinereus. Sin embargo, $\boldsymbol{M}$. cirrosus produce ascosporas ampliamente reniformes de 5-6 x 3-4 $\mu \mathrm{m}$ y grandes conidios, mientras $\boldsymbol{M}$. cinereus produce ascosporas ampliamente reniformes o también triangulares de 4-5,5 x 2,5-4 $\mu \mathrm{m}$, con conidios obovoides a clavados de 3-5 x 2-3 $\mu \mathrm{m}$. Figura A ascosporas: tomada de Krisher et al, (1995), B. Figura tomada de: de Hoog et al, 2000.

5. Ascosporas triangulares en visión plana, 4-5 x 3-4 $\mu \mathrm{m}$ Microascus trigonosporus C.W. Emmons \& B.O. Dodge

(Colonias en MEA de color canela pálido, luego gris ratón, gris oscuro a negro. Ascomata carbonáceos glabros o setosos, 125-200 $\mu \mathrm{m}$ de ancho, ostiolados y con largo cuello, extruyen las ascosporas en largos cirros. Ascos ovados evanescentes, catenados, 10-22 x 7-8 $\mu \mathrm{m}$, ascosporas triangulares en visión plana, con 3 lados redondeados, café rojizas en masa, $4-5,5 \times 3-4 \mu \mathrm{m}$. Conidios globosos a subglobosos a ovoides, de base trunca, redondos a papilados en el ápice, 4-4,5 x 3-4 $\mu \mathrm{m}$. La variedad macrosporus tiene ascosporas de 5,5-7,5 x 5- 6,5 $\mu \mathrm{m}$ y conidios de 5-7 x 4-5 $\mu \mathrm{m}$. Es una especie común del suelo, semillas y excrementos de herbívoros (Barron et al. 1961). Como oportunista en humanos se ha asociado a neumonias en pacientes con compromiso inmune (Mohammedi et al. 2004) y endocarditis (Wang et al. 2011). Entre las especies que producen esporas triangulares (M. alveolaris, M. campaniformis, M. macrosporus y M. pyramidus), M.trigonosporus produce las ascosporas más pequeñas 3-5 x 3-4 $\mu \mathrm{m}$ ). Figuras tomadas de: Matsushima (1975). Para mayores datos en estos géneros, ver (Morton \& Smith, (1963) y Sandoval-Denis, et al, 2015).

\section{REFERENCIAS}

Abbott, S.P.; Sigler, L. \& Currah, R.S. (1998). Microascus brevicaulis sp. nov., the teleomorph of Scopulariopsis brevicaulis, supports placement of Scopulariopsis with the Microascaceae. Mycologia 90: 297-302

Abbott, S.P. \& Sigler, L. (2001). Heterothallism in the Microascaceae demonstrated by three species in the Scopulariopsis brevicaulis series. Mycologia 93:1211-1220

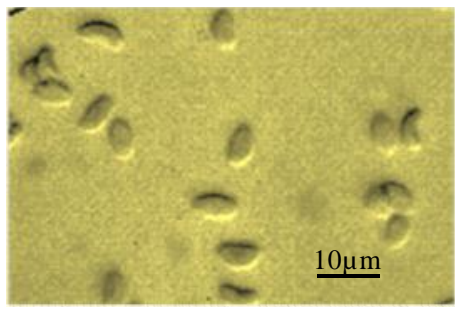

Ascosporas
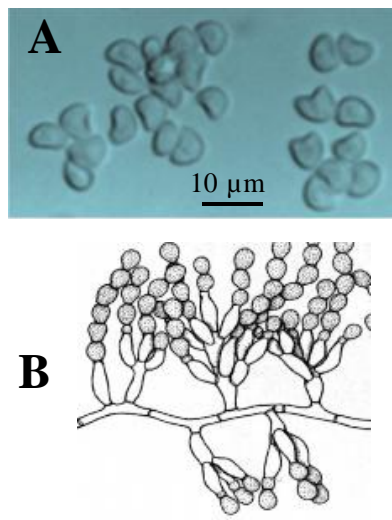

Conidióforos células conidiógenas y conidios

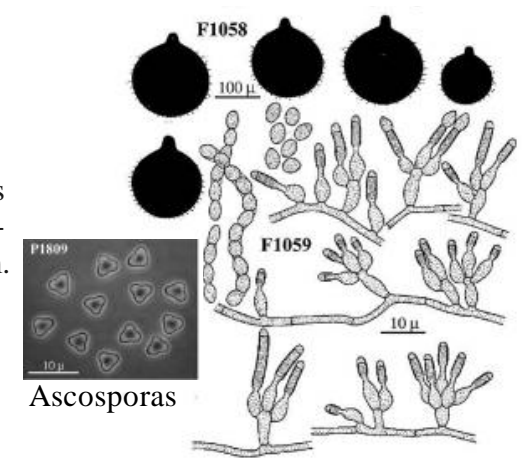

Ascomata, conidióforos, células conidiógenas y conidios
Aguilar C, Pujol I, Guarro J. (1999). In vitro antifungal susceptibilities of Scopulariopsis isolates. Antimicrob. Agents Chemother. 43:1520-1522

Arx, J.A. von. (1973). The genera Petriellidium and Pithoascus (Microascaceae).Persoonia 7:367-375

Arx, J.A. von.; Figueras, M.J. \& Guarro, J. (1988). Sordariaceous ascomycetes without ascospore ejaculation. Beihefte zur Nova Hedwigia 94:1-104 
Baddley, J.W.; Moser, S.A.; Sutton, D.A, et al. (2000). Microascus cinereus (anamorph Scopulariopsis) brain abscess in a bone marrow transplant recipient. Journal of Clinical Microbiology 38:395-397

Barron, G.L.; Cain, R.F.; Gilman, J.C. (1961). The genus Microascus. CanadianJournal of Botany 39:1609-1631

Bentley, Ronald. \& Chasteen, Thomas. G. (2002). «Microbial Methylation of Metalloids: Arsenic, Antimony, and Bismuth». Microbiology and Molecular Biology Reviews 66:250-271

Besbes, M. et al, (2002). Otomicosis debido a Scopulariopsis brevicaulis.Rev. Laryngol. Otol. Rhinol. (Bord). 123:77-8

Biourge, P. (1923). Les moisissures du groupe Penicillium Link: etude monographique. La Cellule 33:7-331

Curzi, M. (1931). Rapporti fra i generi Microascus Zukal e Scopulariopsis Bainier. Bolletino della Stazione di Patologia Vegetale di Roma 11:55-60

de Hoog, G.S.; Guarro, J.; Gené, J.; Figueras, M.J. (2000). Atlas of clinical fungi, 2nd ed. Centraalbureau voor Schimmelcultures, Utrecht, The Netherlands

de Hoog, G.S.; Guarro, J.; Gené, J. et al. 2011. Atlas of clinical fungi. CD-ROM version 3.1. CBS-KNAW Fungal Biodiversity Centre, Utrecht.

Domsch, K.H.; Gams, W.; Anderson, T,H. (2007). Compendium of soil fungi ed 2.Eching, IHW Verlag

Gluck O, Segal N, Yariv F, Polacheck I, et al. (2011). Pediatric invasive sinonasal Scopulariopsis brevicaulis-a case report and literature review. Int. J. Pediatr. Otorhinolaryngol. 75:891-893

Guarro, J.; Gené, J.; Stchigel, A.M. et al. (2012). Atlas of soil ascomycetes. CBS. Biodiversity Series. CBS-KNAW Fungal Biodiversity Centre, Utrecht, The Netherlan

Hawksworth, D.L. (2012). Managing and coping with names of pleomorphic fungi in a period of transition. IMA Fungus 3:15-24

Hennequin, C, et al (1994). Scopulariopsis brevicaulis otomicosis después de timpanoplastia. Ann. Otolaryngol. Chir. Cervicofac. 111: 353-354

Issakainen, J.; Jalava, J.; Hyvönen, J, et al. (2003). Rela-tionships of Scopulariopsis based on LSU rDNA sequences. Medical Mycology 41:31-42

Iwen, P.; Schutte, S.D.; Florescu, D.F. et al. (2012). Invasive Scopulariopsis brevicaulis infection in an immunocompromised patient and review of prior cases caused by Scopulariopsis and Microascus species. Medical Mycology 50:561-569

Krisher, K.K.;Holdridge, N.B.; Mustafá, M.M. et al, (1995). Disseminated Microascus cirrosus Infection in Pediatric Bone Marrow Transplant Recipient.J.Clin.Microbiol. 33:735-737

Kriesel, J.D,et al, (1994). Invasive sinonasal disease due to Scopulariopsis candida: case report and review of Scopulariopsis. Clin. Infect. Dis. 19:317-319
Lumbsch, H.T. \& Huhndorf, S.M. (2007). Outline of Ascomycota 2007. Myconet 13:1-58

Mohammedi, I.; Piens, M.; Audigier-Valette, C. et al, (2004). Fatal Microascu strigonosporus (anamorph Scopulariopsis) pneumonia in a bone marrow transplant recipient. European Journal of Clinical Microbiology \& Infectious Diseases 23:215-217

Matsushima, T. (1975). Icones Microfungorum un Lectorum Matsushima. : 1-209

Migrino, R.Q.; Hall, G.S,. \& Longworth, D.L. (1995). Deep tissue infectionscaused by Scopulariopsis brevicaulis: report of a case of prosthetic valve endocarditis and review. Clin.Infect.Dis. 21:672-674

Miossec, C. et al, (2011). Fatal Invasive Infection with Fungemia Due to Microascus cirrosus after Heart and Lung Transplantation in a Patient with Cystic Fibrosis. J. Clin. Microbiol. 49:2743-2747

Morton, F.J. \& Smith, G. (1963). The genera Scopulariopsis Bainier, Microascus Zukal, and Doratomyces Corda. Mycological Papers 86:1-96

Patel, R. et al, (1993). Phaeohyphomycosis due to Scopulariopsis brumptii in a liver transplant recipient. Clin. Infect. Dis. 19:198-200

Petit A, et al, (2011). Scopulariopsis brevicaulis abscess in a child treated for myeloblastic leukaemia. Lancet Infect. Dis. 11:416

Piontelli, E. \& Toro, M.A. (1988).Comentarios biomorfológicos y clínicos sobre el génerop Scopulariopsis Bainier. Hialohifomicosis en uñas y piel. Boletín Micológico 3:259-273

Ragge, N.K;, Hart,. JC.; Easty, D.L. \& Tyers, A.G. (1990). A case of fungal keratitis caused by Scopulariopsis brevicaulis: treatment with antifungal agents and penetrating keratoplasty. Br. J. Ophthalmol. $74: 561-562$

Revankar, S.G. \& Sutton, D.A. (2010). Melanized Fungi in Human Disease.Clin. Microbiol. Rev. 23:884-928

Ropars, J.; Cruaud, C.; Lacoste, S, et al. (2012). A taxonomic and ecological overview of cheese fungi. International Journal of Food Microbiology 155:199-210

Saccardo, P.A. (1882). Fungi veneti novi vel critici vel mycologiae venetae addendi. Series 13. Michelia 2:528-563

Sandoval-Denis, M.; Sutton, D.A.; Fothergill, A.W, et al. (2013) Scopulariopsis, apoorly known opportunistic fungus: spectrum of species in clinical samples and in vitro responses to antifungal drugs. Journal of Clinical Microbiology 51: 3937-3943

Sandoval-Denis, M.; Gene,J.; Sutton,D.A. et al, (2015). Redefinining Microascus, Scopulariopsis and allied genera. Persoonia 36:1-36

Sopp, O.J. (1912). Monographie der Pilzgruppe Penicillium mit besonderer Berücksichtigung der in Norwegen gefunden Arten. Videnskaps Selskapets Skrifter 1. Matematisk-Naturvidenskabelig Klasse 11: $1-207$

Wang, P.; Wang, H.; Zhao, Y. et al. (2011). A case of endocarditis caused by Microascus trigonosporus. Chinese Journal of Mycology 6:162-165 